\title{
Solving the puzzle of foothill abortion in beef cattle
}

\author{
by Glenn A. Nader, Mike N. Oliver, Julie A. Finzel, Myra T. Blanchard and Jeff L. Stott
}

Eooth oothill abortion, also known as epizootic bovine abortion (EBA), has been a long-standing problem for California beef cattle producers. It is a major source of economic loss for California cow and calf producers, and in the 1990s it was estimated that $5 \%$ to $10 \%$ ( 45,000 to 90,000 calves) of the California beef calf crop may be lost each year (Bushnell et al. 1991). UC Cooperative Extension (UCCE) farm advisors, specialists and UC Davis School of Veterinary Medicine (SVM) faculty have worked on this disease for nearly 50 years. This long research process finally moved forward in 2005, when the causative agent was identified.

The Pajaroello (pa-ha-WAY-lo) tick, Ornithodoros coriaceus, is responsible for transmitting the causative agent (a deltaproteobacterium) when it feeds on a pregnant cow. The Pajaroello is a soft-bodied tick that resides in dirt or litter under trees and bushes, locations where deer and cattle typically bed down. The Pajaroello does not embed itself in animal flesh, but rather it feeds rapidly (for as little as 20 minutes) and then drops back onto the ground. It can survive for years in a dormant state, without taking a blood meal.

If a cow or heifer is bitten by a tick when 2 to 6 months pregnant, the calf may abort or be born weak. Heifers and cows that have not previously grazed in tick-infested pastures are most susceptible. Once bitten, cows appear to gain some degree of immunity, but ranchers have observed that immunity can be lost if cattle go for a year or more without tick bites, which serve as an immunity booster.

Early UC efforts focused on identifying the vector of the disease. First, mosquitoes were suspected. They were eliminated as a possibility when cattle elevated off the ground (in an area where the disease commonly occurred) carried their calves to term. Additional experiments also eliminated the Leptoconops gnat as a possible vector.

It was initially thought that the Pajaroello tick did not live in the most northern areas of California, where EBA occurred. When ticks were subsequently trapped on a northern Lassen County ranch that had experienced abortions, it was confirmed as a potential vector. Ticks were collected and placed to feed on susceptible heifers (on the UC Davis campus), and abortions occurred, confirming the Pajaroello as the vector of the disease.

The next step was to determine the causative agent being transmitted by the bite of the tick. This was difficult because the tick harbors numerous potential causative agents. In the late 1960s and through the 1970s, chlamydia was considered as a possible causative agent. During this period, a field trial was conducted with cooperator cattle in Lassen County in which susceptible heifers were fed tetracycline crumbles, and the data suggested there might be some protection from the antibiotics. Numerous chlamydia vaccines were prepared and given to susceptible heifers, but this effort was ultimately abandoned when heifers continued to abort following vaccination.

In the 1970s and early 1980s, viruses were considered as possible causative agents. A large research effort was initiated, with over 80 viruses isolated from the tick. After exhaustive work, research on causative agents moved from viruses to spirochete-like organisms; a Borrelia species was suggested to be a potential cause of EBA, but further experimental studies essentially eliminated spirochetes and Borrelia species as potential causative agents.

With no definitive causal agent of EBA identified, the California Cattlemen's Association gave UCCE a grant from its Livestock

Memorial Research Fund to develop educational outreach through a video on how to manage cattle to minimize the impact of the disease. Farm advisors and specialists with knowledge of the tick's feeding habitats and how the abortions developed in cattle used case studies with ranchers to develop management options that ranchers could use to lessen the impact of the disease on their business. Successful practices included preexposing sexually mature heifers to known tick areas prior to breeding, avoiding tick areas during the critical 2 to 6 months of pregnancy and shifting from spring to fall calving in the most northern regions of the state.

SVM researchers and the California Animal Health and Food Safety (CAHFS) laboratory system, using a large number of aborted calves, were able to develop methods to identify foothill abortion in aborted calves. This knowledge was extended to practicing veterinarians working with ranches throughout the state.

In 2002, a SVM laboratory developed a reliable challenge system for experimental transmission of EBA that was used to establish that the causative agent was antibiotic susceptible. This report was quickly followed with a positive identification of the agent causing foothill abortion, a bacteria belonging to a very unusual group of slime bacteria; then referred to simply as the agent of EBA, the bacterial pathogen has now been unofficially named Pajaroellobacter abortibovis. 
Other breakthroughs followed quickly. The cultivation of the bacteria in immunodeficient mice gave new life to research efforts. A vaccine development phase was initiated with over $\$ 200,000$ from the California Cattlemen Association's Livestock Memorial Research Fund and financial support from SVM and their collaborators at the University of Nevada, Reno. In 2009, a small group of heifers were protected against experimental infection after they were immunized several weeks prior to breeding with a candidate vaccine that was both live and infectious.

The success of a second and larger trial in 2010 prompted SVM researchers to pursue product licensing with the U.S. Department of Agriculture (USDA) Center for Veterinary Biologics. Vaccine efficacy experiments were conducted in accordance with USDA regulations. University-owned heifers were immunized before breeding and then administered an artificial challenge with virulent bacteria at the peak of fetal susceptibility (100 days gestation). Vaccine field trials that combined USDA-required field safety trials with field efficacy were then initiated at the UC Sierra Foothill Research and Extension Center on UC Davis Department of Animal Science heifers, on heifers at University of Nevada, Reno, and on producer-owned beef herds in California and Nevada. Over 1,600 heifers were enrolled in these trials in 2011 and 2012. Additional funding for such a massive effort was provided to the SVM by UC's Office of the President via a UC Proof of Concept Discovery Grant (grant ID no. 212263).

Although the results of these studies are currently being assembled, preliminary assessment of the experimental vaccine indicates excellent protection against foothill abortion has been successfully demonstrated. All the successes realized to date were a result of SVM collaborations across the UC spectrum with CAHFS's diagnostic laboratory at UC Davis, the UC Davis Department of Animal Science, UCCE, the Sierra Foothill Research and Extension Center, and also with researchers at the University of Nevada, Reno.

Historically, specialty vaccines created for use only in California were licensed through the California Department of Food and Agriculture (CDFA). Currently, CDFA does not process new specialty vaccines, requiring researchers to work with the USDA to get the foothill abortion vaccine licensed. USDA requirements are more stringent than CDFA's requirements, as food animal vaccines must comply with the federal Virus-Serum-Toxin Act requirements. SVM and USDA are charting new territory as they work to certify the safety and efficacy of the vaccine. The developers of the vaccine at SVM are in the process of establishing a USDA-required vaccine seed, determining if production can be scaled up to a commercial level and identifying viable options for commercial production of the vaccine.

As the commercialization efforts proceed, researchers are finetuning the vaccination regime to address concerns over the prolonged persistence of the vaccine bacteria and the potential impact

\section{References}

Blanchard MT, Chen Cl, Anderson M, et al. 2010. Serial passage of the etiologic agent of epizootic bovine abortion in immunodeficient mice. Vet Microbiol 144(1-2):177-82.

Bushnell R, Oliver M, Nader G, Norman B. 1991. Foothill Abortion. Veterinary Medicine Extension, Cooperative Extension, School of Veterinary Medicine, University of California, Davis.

King DP, Chen C-I, Blanchard MT, et al. 2005. Molecular identification of a novel deltaproteobacterium as the etiologic agent of epizootic bovine abortion (foothill abortion). J Clin Microbiol 43(2):604-9.

Stott JL, Blanchard MT, Anderson M, et al. 2002. Experimental transmission of epizootic bovine abortion (foothill abortion). Vet Microbiol 88(2):161-73. on embryonic mortality in animals bred within weeks following vaccination. These studies are being conducted using a combination of UC and private producer replacement heifers. The vaccine dose is being adjusted downward, and the time from vaccination to breeding is being extended. The vaccine cannot be administered to pregnant cattle. Skin reactions following vaccination suggest that the live bacterial pathogen can persist for up to 2 months. On the positive side, this bacterial persistence induces a solid immunity that likely lasts through the next breeding cycle and possibly beyond. Studies are under way to begin to address length of immunity.

The fact that the vaccine is live and infectious poses several unique challenges. For example, the cryopreserved bacteria must be transported and stored in liquid nitrogen, and the cost of purchasing the vaccine could also be high because of the cost of manufacturing - the live vaccine must be cultured in an immunodeficient mouse. The California Cattlemen's Association is working to develop a regional distribution system for the vaccine, which could become available within a couple of years.

Work is being conducted to develop a recombinant vaccine through genomic research. In a recombinant vaccine, the genomic sequence of candidate bacterial genes must first be established. Next, the genes must be expressed as protein and then combined with adjuvant(s) to construct candidate vaccines. A recombinant vaccine would be far less sensitive to temperature and would not require immunodeficient mice in the manufacturing process, thereby making the finished product much more cost effective and practical for on-ranch use.

Subsequent findings by the SVM researchers have also improved the diagnostic procedures for identifying foothill abortions at the CAHFS diagnostic laboratory at UC Davis and have provided additional important information for UCCE to extend to ranchers to confirm abortions caused by the disease. Researchers are now actively pursuing validation of a diagnostic assay that may allow ranchers in the future to identify cows that have been exposed to the tick. Such an assay could be used to establish susceptibility of naïve replacement heifers to foothill abortion or confirm whether the disease is present on a ranch.

Decades of hard work by UC researchers and educators all across the system have allowed the pieces of this difficult disease puzzle to come together in assisting California's cattle ranchers. CA

G.A. Nader is UC Cooperative Extension (UCCE) Livestock and Natural Resources Farm Advisor, Sutter, Yuba and Butte counties; M.N. Oliver is UCCE StaffResearch Assistant, Retired; J.A. Finzel is UCCE Livestock and Natural Resources Advisor, Kern, Kings and Tulare counties; M.T. Blanchard is Specialist in Pathology, Microbiology and Immunology, UC Davis School of Veterinary Medicine; and J.L. Stott is Professor of Pathology, Microbiology and Immunology, UC Davis School of Veterinary Medicine.

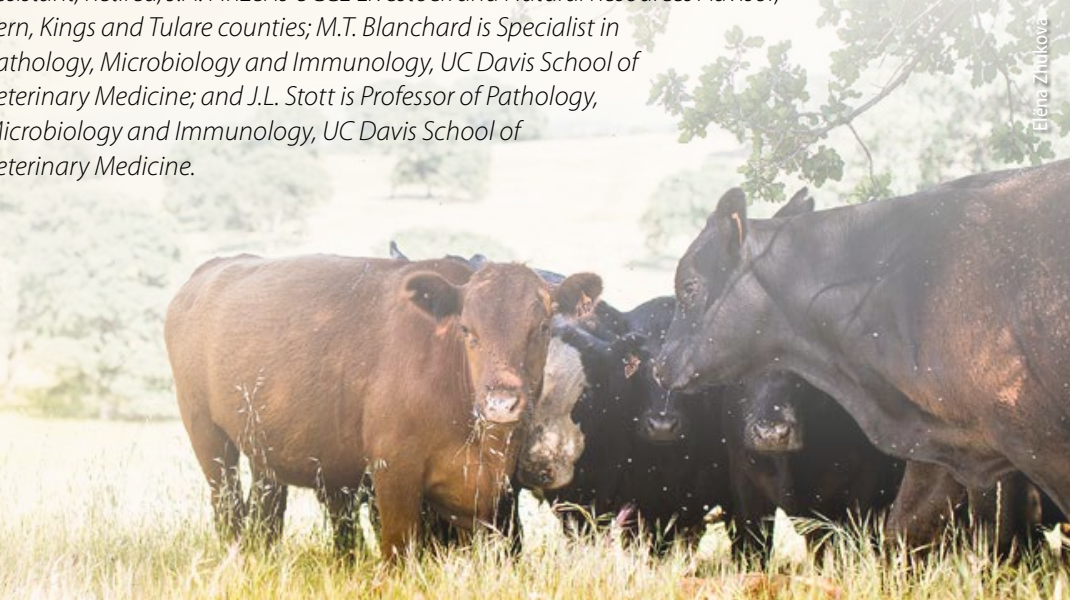

